\title{
Análise de critérios do processo de curadoria de RED em plataformas digitais: uma perspectiva comparativa
}

\author{
Alexandre Ladvig ${ }^{1}$, Ana Leticia Oliveira do Amaral ${ }^{2}$, Julia Maria Gerhardt da \\ Rocha $^{1}$, Marina Bazzo de Espíndola ${ }^{3}$, Roseli Zen Cerny ${ }^{3}$, Diego França Vieira ${ }^{1}$ \\ ${ }^{1}$ Laboratório de Novas Tecnologias - Universidade Federal de Santa Catarina (UFSC) - \\ Florianópolis, SC - Brasil \\ ${ }^{2}$ Programa de Pós-Graduação em Design - Universidade Federal de Santa Catarina \\ (UFSC) - Florianópolis, SC - Brasil \\ ${ }^{3}$ Programa de Pós-Graduação em Educação Científica e Tecnológica - Universidade \\ Federal de Santa Catarina (UFSC) - Florianópolis, SC - Brasil \\ ${ }^{4}$ Programa de Pós-Graduação em Educação - Universidade Federal de Santa Catarina \\ (UFSC) - Florianópolis, SC - Brasil \\ \{alexandreladvig, amaral.analeticia, juliagerhardt.rocha, marinabazzo, \\ rosezencerny, dfvdiego\}@gmail.com
}

\begin{abstract}
Resumo. O presente estudo se insere no contexto de um projeto de pesquisa e desenvolvimento de uma Plataforma Educacional Digital que reúne e disponibiliza recursos educacionais digitais (RED). Nosso objetivo é identificar e analisar os critérios utilizados em diferentes tipos de sistemas de curadoria de recursos educacionais digitais presentes em plataformas similares a uma Plataforma Digital específica. Esta investigação deu-se a partir das concepções de curadoria e estudos referente ao ciclo de vida de um RED e uma matriz de referência sobre possíveis avaliações de um RED. Entendemos que o processo de Curadoria Digital acontece em momentos diferentes e ao longo de todas as etapas do ciclo de vida de um RED.
\end{abstract}

\begin{abstract}
The present study is part of the context of a research and development project of a Digital Educational Platform that gathers and provides digital educational resources (DER). Our aim is to identify and analyze the criteria used in different types of curatorship systems of digital educational resources present on platforms similar to a specific Digital platform. This investigation was based on the conceptions of curatorship and studies related to the life cycle of a DER and a reference matrix on possible assessments of a DER. We understand that the Digital curatorial process takes place at different times and throughout all stages of the life cycle of a DER.
\end{abstract}

\section{Introdução}

Este estudo faz parte de um projeto maior, de pesquisa e desenvolvimento de uma plataforma que reúne e dispõe os Recursos Educacionais Digitais (RED) dos principais 
portais educacionais do Brasil como, por exemplo, Portal do Professor, Banco Internacional de Objetos Educacionais (BIOE), Domínio Público e Portal TV Escola. A Plataforma Integrada MEC RED, foi desenvolvida e implementada em uma parceria entre as Universidades Federais de Santa Catarina (UFSC) e do Paraná (UFPR) a partir de uma demanda da Secretaria de Educação Básica e do Ministério da Educação. Ao longo de todo o processo, buscou-se a participação de professoras(es) da Educação Básica de todo o Brasil. Os educadores tiveram e têm papel ativo no desenvolvimento da plataforma e o projeto procurou compreender e trazer as realidades desses profissionais - tanto dentro, quanto fora da sala de aula - no exercício de uma abordagem de design mais colaborativo e participativo. A plataforma está disponibilizada no website ${ }^{1}$ desde Novembro de 2017.

Segundo Barros (2014) a curadoria digital envolve dois componentes diferentes e relacionados: a curadoria por intervenção humana; e por algoritmo curador, sendo que eles não são dissociáveis. Partindo de reflexões teóricas acerca de curadoria, Abbott (2008), Sales e Sayão (2012) concluem que este processo serve como uma garantia de conservação de dados sem que percam seu valor. Entendemos, assim, a importância de efetivar a curadoria digital da maneira mais precisa possível e a necessidade de propor um modelo de curadoria para a Plataforma Integrada MEC de Recursos Educacionais Digitais.

Para isso, compreendemos a necessidade de explorar e analisar os processos de curadoria de diferentes plataformas que tenham semelhança com a Plataforma MEC RED para então realizarmos uma proposta coletiva fundamentada empiricamente. Nesse sentido, o presente estudo tem como objetivo identificar e analisar os critérios utilizados em diferentes tipos de sistemas de curadoria de recursos educacionais digitais presentes em plataformas similares a Plataforma Integrada MEC RED. Buscamos, dessa maneira, subsidiar uma futura proposta de curadoria para a Plataforma que atenda aos requisitos do projeto, bem como esteja em consonância com as melhores práticas adotadas no contexto de plataformas que disponibilizam RED. Esperamos também contribuir com a discussão acerca de modelos e metodologias de curadoria digital de RED.

\section{Fundamentação Teórica}

Esta investigação baseia-se nas concepções de curadoria digital, que envolve a seleção, avaliação e organização de um conjunto de mídias de qualquer natureza, para as mais diversas práticas contemplando as esferas de atividade sociais diversificadas. O termo vem sendo usado com frequência como "as ações necessárias para manter dados de pesquisa em meio digital e outros materiais ao longo de seus ciclos de vida e do tempo para as gerações atuais e futuras de usuários" [Beagrie 2004, p.7]. Além disso, segundo Barros (2014) o processo de curadoria digital envolve, dois segmentos distintos e interligados: o algoritmo curador e a intervenção humana. A autora ainda defende que o processo de curadoria digital não se limita pelo algoritmo curador, entendendo que o processo "necessita de discernimento e de habilidades humanas que vão além da capacidade técnica das máquinas" [Barros 2014, p.34].

\footnotetext{
${ }^{1}$ Para conhecer a Plataforma MEC RED, acesse: https://plataformaintegrada.mec.gov.br/
} 
VIII Congresso Brasileiro de Informática na Educação (CBIE 2019)

Anais do XXX Simpósio Brasileiro de Informática na Educação (SBIE 2019)

De acordo com Cechinel (2017) o processo de curadoria pode ocorrer nas diferentes fases do ciclo de vida dos RED. Baseado em Collis e Strijker (2004), Cechinel (2017) afirma que o ciclo de vida de um RED no processo de curadoria percorre seis etapas: 1) Obtenção ou Criação, diz respeito ao desenvolvimento do recurso, ou adaptação de outro recurso existente; 2) Etiquetamento, trata da descrição do RED que auxilia no processo de busca, sendo que as informações podem ser básicas (como título ou assunto) ou utilizar padrões mais completos; 3) Oferta, consiste no armazenamento e/ou publicação do RED; 4) Seleção, escolha do recurso pelo usuário; 5) Uso, integração do RED no contexto do usuário e 6) Retenção, etapa onde o RED pode ser revisado, modificado e avaliado, ou até mesmo descontinuado.

A avaliação de recursos educacionais digitais, que está intimamente ligada com a sua seleção, é considerada uma tarefa complexa que necessita de diversos critérios que devem ser observados [Cechinel 2017; Brasil 2019]. Nessa perspectiva, ainda apoiados no estudo de Cechinel (2017), que discorre sobre uma visão geral do funcionamento do processo de curadoria em repositórios, buscamos a tipificação de Hylén (2006) trazida pelo autor. A matriz que classifica possíveis formas de avaliação de RED, contempla quatro campos com dois eixos principais, relacionados com as características de avaliação e de funcionamento. O eixo horizontal compreende a noção de avaliação descentralizada versus centralizada e o eixo vertical abrange a avaliação aberta versus avaliação fechada, figura 1 .

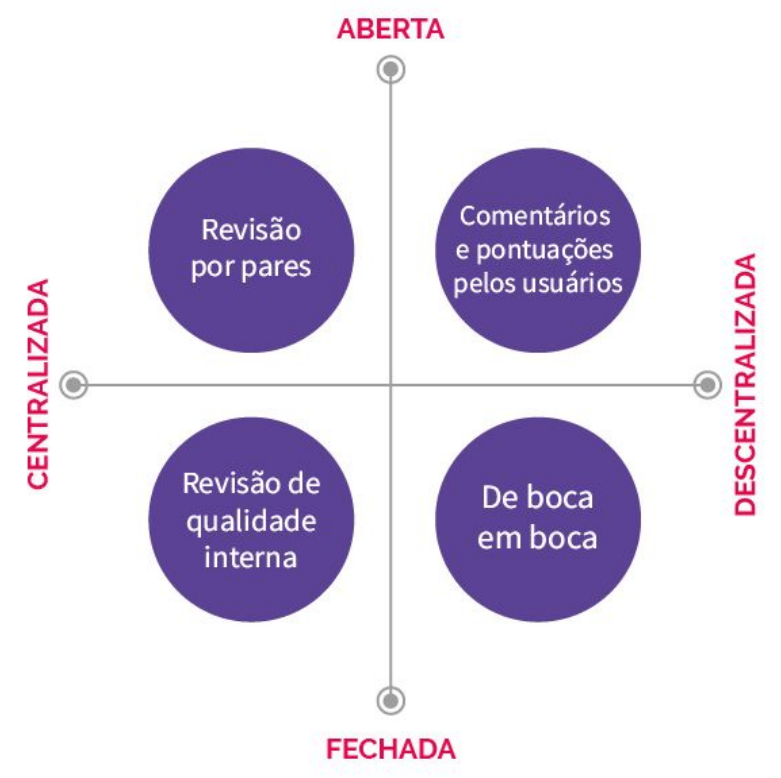

Figura 1. Processo de gestão da qualidade para avaliação de RED, adaptado de Cechinel (2017) e Hylén (2006)

O eixo que traz as concepções de avaliação aberta versus fechada refere-se à transparência do processo de avaliação. O processo é aberto quando as avaliações e os seus critérios são visíveis a todos, do contrário, entende-se como avaliação fechada. $\mathrm{O}$ eixo que aborda as concepções de avaliação centralizada versus descentralizada está relacionado ao controle do processo de quem faz a curadoria. Quando o controle do processo está restrito às plataformas/instituições entende-se que a avaliação é 
VIII Congresso Brasileiro de Informática na Educação (CBIE 2019)

Anais do XXX Simpósio Brasileiro de Informática na Educação (SBIE 2019)

centralizada, como explica Cechinel (2017, p. 12) "a garantia da qualidade é assegurada pelo conhecimento de especialistas e por um conjunto de critérios de avaliação previamente estabelecidos para a condução do processo de avaliação". E, por outro lado, quando o controle está nas mãos da comunidade - em geral os usuários das plataformas -, esses sujeitos auxiliam na definição da qualidade dos recursos por meio de interações entre plataforma e usuário (comentários, pontuações entre outros) relacionados às impressões de uso dos mesmos em seus contextos, considera-se a avaliação descentralizada.

Cabe ressaltar que avaliação por especialistas de forma descentralizada ou avaliação dos usuários de forma centralizada são possibilidades pouco prováveis, pois suas definições são baseadas na maneira como a avaliação é concedida. Portanto, se a avaliação é realizada por especialistas, de fato ela é centralizada. Ou, se a avaliação é feita pela comunidade usuária ela é descentralizada.

\section{Metodologia da pesquisa}

Neste estudo foi desenvolvida uma análise exploratória dos critérios pertencentes ao processo de curadoria digital de RED de diferentes plataformas educacionais digitais. Buscamos, também, alcançar reflexões referente aos diferentes processos analisados.

No campo de estudo do design, a análise de similares permite, segundo Gomes (2004), verificar o produto em seu contexto e relacioná-lo aos seus semelhantes . Dessa maneira, Meurer e Szabluk (2010) recomendam que a análise de similares se inicia a partir da escolha de um número razoável de produtos (entre 10 a 20), para então identificar, apresentar e descrever os pontos positivos e negativos de cada um.

Dessa maneira, a escolha das plataformas analisadas, se deu a partir de três principais frentes. Primeiramente foram considerados as plataformas governamentais que estão integradas ${ }^{2}$ à Plataforma MEC RED: Escola Digital, TV Escola, Banco Internacional de Objetos Educacionais (BIOE) e Portal do Professor. Posteriormente, selecionamos 2 plataformas encontradas em 3 artigos sobre curadoria que compõe o referencial teórico deste estudo, 4 indicadas por pesquisadores parceiros e 3 por meio do evento Open Education Week $^{3}$, que busca anualmente conscientizar e mostrar o impacto da educação aberta no ensino e aprendizagem, através da divulgação de eventos presenciais e webinars, projetos e recursos educacionais. Ao total, selecionamos 14 plataformas apresentadas no quadro 1.

Quadro 1. Plataformas analisadas

\begin{tabular}{|c|c|c|c|}
\hline $\begin{array}{l}\text { Nome da } \\
\text { Plataforma }\end{array}$ & Descrição & $\begin{array}{l}\text { Link para } \\
\text { acesso }\end{array}$ & Iniciativa \\
\hline $\begin{array}{c}\text { Banco } \\
\text { Internacional } \\
\text { de Objetos } \\
\text { Educacionais } \\
\text { (BIOE) }\end{array}$ & $\begin{array}{l}\text { Disponibiliza recursos educacionais gratuitos } \\
\text { em diversas mídias e idiomas que atendem } \\
\text { desde a educação básica até a superior. }\end{array}$ & $\begin{array}{l}\underline{\text { http://objetosedu }} \\
\underline{\text { cacionais.mec.g }} \\
\underline{\text { ov.br }}\end{array}$ & $\begin{array}{l}\text { Governamental } \\
\text { Ministério da } \\
\text { Educação (Brasil) }\end{array}$ \\
\hline
\end{tabular}

\footnotetext{
${ }^{2}$ A plataforma Domínio Público, apesar de estar integrada à Plataforma MEC RED, foi desconsiderada da análise por possibilitar apenas download dos recursos.

${ }^{3}$ https://www.openeducationweek.org/
} 
VIII Congresso Brasileiro de Informática na Educação (CBIE 2019)

Anais do XXX Simpósio Brasileiro de Informática na Educação (SBIE 2019)

\begin{tabular}{|c|c|c|c|}
\hline TV Escola & $\begin{array}{l}\text { Pode ser utilizada como uma ferramenta } \\
\text { pedagógica tanto para formação } \\
\text { complementar do professor, quanto para } \\
\text { utilização em práticas de ensino. }\end{array}$ & $\begin{array}{l}\underline{\text { https://tvescola. }} \\
\underline{\text { org.br/ }}\end{array}$ & $\begin{array}{l}\text { Governamental } \\
\text { Ministério da } \\
\text { Educação (Brasil) }\end{array}$ \\
\hline $\begin{array}{l}\text { Escola } \\
\text { Digital }\end{array}$ & $\begin{array}{l}\text { É uma plataforma gratuita e aberta de busca } \\
\text { de objetos e recursos digitais voltados a } \\
\text { apoiar processos de ensino e aprendizagem } \\
\text { dentro e fora da sala de aula. }\end{array}$ & $\underline{\underline{\text { https://rede.esco }}}$ & Privada \\
\hline $\begin{array}{l}\text { Portal do } \\
\text { Professor }\end{array}$ & $\begin{array}{l}\text { Tem como objetivo apoiar os processos de } \\
\text { formação dos professores brasileiros e } \\
\text { enriquecer a sua prática pedagógica. }\end{array}$ & $\begin{array}{l}\text { http://portaldopr } \\
\text { ofessor.mec.gov } \\
\underline{\text { br/index.html }}\end{array}$ & $\begin{array}{l}\text { Governamental } \\
\text { Ministério da } \\
\text { Educação (Brasil) }\end{array}$ \\
\hline Merlot & $\begin{array}{l}\text { Fornece acesso a materiais de aprendizagem } \\
\text { e suporte online com curadoria e ferramentas } \\
\text { de criação de conteúdo, liderados por uma } \\
\text { comunidade internacional de educadores, } \\
\text { alunos e pesquisadores. }\end{array}$ & $\begin{array}{l}\underline{\text { https://www.mer }} \\
\underline{\text { lot.org/merlot/in }} \\
\underline{\text { dex.htm }}\end{array}$ & $\begin{array}{l}\text { Governamental } \\
\text { Universidade da } \\
\text { Califórnia, } \\
\text { voluntários e } \\
\text { membros. }\end{array}$ \\
\hline $\begin{array}{c}\text { OER } \\
\text { Commons }\end{array}$ & $\begin{array}{l}\text { É uma biblioteca digital pública de recursos } \\
\text { educacionais abertos. Explore, crie e } \\
\text { colabore com educadores de todo o mundo } \\
\text { para melhorar o currículo. }\end{array}$ & $\frac{\text { https://www.oer }}{\underline{\text { commons.org/ }}}$ & $\begin{array}{l}\text { Organização sem } \\
\text { fins lucrativos - } \\
\text { Estudo da Gestão } \\
\text { do Conhecimento } \\
\text { na Educação } \\
\text { (ISKME) }\end{array}$ \\
\hline Entramar & $\begin{array}{l}\text { A plataforma faz parte de um programa de } \\
\text { inovação educacional da secretaria de } \\
\text { educação do município de Vicente } \\
\text { López/Argentina. }\end{array}$ & $\frac{\underline{\text { https://www.entr }}}{\underline{\text { amar.mvl.edu.ar }}}$ & $\begin{array}{l}\text { Governamental } \\
\text { Secretaria da } \\
\text { Educação } \\
\text { (Argentina) }\end{array}$ \\
\hline Relia & $\begin{array}{l}\text { O REliA é uma plataforma que organizar o } \\
\text { levantamento de recursos com licenças } \\
\text { abertas feito pelo Instituto Educadigital via } \\
\text { projeto REA.br }\end{array}$ & $\underline{\text { https://relia.org. }}$ & $\begin{array}{l}\text { Governamental } \\
\text { Iniciativa } \\
\text { Educação Aberta } \\
\text { (Brasil) }\end{array}$ \\
\hline REA Ceibal & $\begin{array}{l}\text { Repositório de recursos educacionais aberto, } \\
\text { os recursos são gerenciados por um equipe } \\
\text { formada por professores do Ensino } \\
\text { Fundamental e Médio. }\end{array}$ & $\begin{array}{l}\text { https://rea.ceibal } \\
\underline{\text { edu.uy/ }}\end{array}$ & $\begin{array}{l}\text { Governamental } \\
\text { Departamento de } \\
\text { Treinamento do } \\
\text { Plano Ceibal } \\
\text { (Uruguai) }\end{array}$ \\
\hline $\begin{array}{l}\text { Common } \\
\text { Sense } \\
\text { Education }\end{array}$ & $\begin{array}{l}\text { É uma organização sem fins lucrativos que } \\
\text { analisa livros, filmes, programas de TV, } \\
\text { jogos de vídeo, aplicativos, músicas e sites e } \\
\text { classifica-os em termos de conteúdo } \\
\text { educacional adequado. }\end{array}$ & $\begin{array}{l}\underline{\text { https://www.co }} \\
\underline{\text { mmonsense.org/ }} \\
\underline{\text { education/ }}\end{array}$ & $\begin{array}{l}\text { Associação } \\
\text { Americana entre } \\
\text { fundações } \\
\text { nacionais, } \\
\text { internacionais, } \\
\text { corporativas, } \\
\text { familiares e locais. }\end{array}$ \\
\hline EdModo & $\begin{array}{l}\text { Empresa de tecnologia educacional que } \\
\text { oferece uma plataforma de comunicação, } \\
\text { colaboração e treinamento para escolas e } \\
\text { professores do ensino fundamental e médio. }\end{array}$ & $\frac{\text { https://www.ed }}{\underline{\text { modo.com/ }}}$ & Privada \\
\hline
\end{tabular}


VIII Congresso Brasileiro de Informática na Educação (CBIE 2019)

Anais do XXX Simpósio Brasileiro de Informática na Educação (SBIE 2019)

\begin{tabular}{|c|c|c|c|}
\hline Curriki & $\begin{array}{l}\text { O modelo de Curriki é desenvolver } \\
\text { currículos por meio de colaboradores da } \\
\text { comunidade e distribuir currículos e recursos } \\
\text { educacionais abertos globalmente. }\end{array}$ & $\underline{\text { https://www.cur }}$ & Governamental \\
\hline $\begin{array}{l}\text { Amazon } \\
\text { Inspire }\end{array}$ & $\begin{array}{l}\text { Permite descobrir, gerenciar, avaliar, revisar } \\
\text { e compartilhar recursos educacionais. }\end{array}$ & $\frac{\underline{\text { https://www.am }}}{\underline{\text { azoninspire.com }}}$ & Privada \\
\hline CK-12 & $\begin{array}{l}\text { Oferece aprendizagem gratuita e } \\
\text { personalizada para todos os alunos de } \\
\text { diferentes níveis da educação. Há a } \\
\text { possibilidade de criar salas de aula digitais, } \\
\text { personalizar livros didáticos e aprender } \\
\text { conceitos de STEM K-12. }\end{array}$ & $\frac{\underline{\text { https://www.ck1 }}}{\underline{\text { 2.org/student/ }}}$ & $\begin{array}{l}\text { A CK-12 } \\
\text { Foundation é uma } \\
\text { organização sem } \\
\text { fins lucrativos }\end{array}$ \\
\hline
\end{tabular}

A análise foi realizada considerando as especificidades do ciclo de vida dos RED [Cechinel 2017], bem como a tipificação das dimensões de avaliação de recursos educacionais dessa natureza [Hylén 2006]. Apesar das diferenças estruturais das plataformas, foi possível uma análise acerca de seus pontos de encontro, bem como dos seus distanciamentos. Como resultado, tivemos dados assertivos sobre como as dimensões e critérios se aplicam ao processo de curadoria de RED e sobre a forma como se configuram estes sistemas de avaliações.

\section{Resultados e discussões}

Para fins de padronização dos instrumentos de análise, a consulta às plataformas deu-se em duas etapas principais, primeiramente os pesquisadores efetuaram uma navegação livre para fins de familiarização com o sistema. Já a segunda etapa consistiu em uma navegação guiada a partir da adaptação do referencial sobre o ciclo de vida de RED com os seguintes passos: a) cadastramento na plataforma, b) leitura das políticas do conteúdo e/ou termos de uso, c)publicação de um RED pré definido, d) busca de recurso e e) avaliação de um RED.

Assim, foi possível perceber algumas especificidades presentes no processo de curadoria digital de tais plataformas. Dessa forma, o processo de análise foi estruturado em dois principais momentos temporais: durante a disponibilização do recurso e a ação após à publicação do recurso. O primeiro momento, disponibilização do recurso, trata sobre a funcionalidade de envio do recurso por parte dos usuários, bem como do processo de verificação de entrada que as plataformas adotam para selecionar e disponibilizar os RED. E o segundo, após a publicação, engloba a maneira que a qualidade dos recursos presentes nas plataformas são atestados, ou seja, o momento das avaliações (sejam elas feitas por especialistas e/ou pela comunidade de usuários).

No primeiro momento, durante a publicação do recurso, a análise concentrou-se em delinear cinco aspectos: a criação; o etiquetamento; a avaliação da submissão; a política de conteúdo e as formas de armazenamento do RED, sendo alguns aspectos já mencionados no ciclo de vida dos RED (CECHINEL, 2017). Entendemos os aspectos como:

- Criação: refere-se a quem tem a autorização para submeter os recursos à plataforma; 
VIII Congresso Brasileiro de Informática na Educação (CBIE 2019)

Anais do XXX Simpósio Brasileiro de Informática na Educação (SBIE 2019)

- Etiquetamento: detalha os metadados utilizados no recurso;

- Avaliação da submissão: atenta para a maneira que as plataformas captam estes arquivos, e se há um processo de verificação dos recursos antes de disponibilizá-los na plataforma;

- Políticas de conteúdo: se refere às diretrizes às quais os recursos presentes na plataforma devem estar alinhados (ex: não conter conteúdo de propaganda, não haver pornografia e possuir a licença creative commons);

- Armazenamento: reflete as questões estruturais dos portais quanto à disponibilização dos conteúdos, nesse caso se disponibilizam por redirecionamento através de links ou se armazenam o recurso dentro da própria plataforma. O termo armazenamento é semelhante ao item "oferta" mencionado por Cechinel (2017), contudo optou-se pela troca do nome, para não haver confusões com o ato de publicar.

Dessa maneira, percebeu-se que, as plataformas Merlot, Curriki e CK-12 não possuem verificação de entrada para a disponibilização de RED, qualquer recurso pode ser adicionado livremente. As plataformas TV Escola, BIOE, Relia, REA Ceibal, Common Sense Education não possuem esta funcionalidade. Já as plataformas Escola Digital, Portal do Professor, OER Commons, Entramar, Edmodo e Amazon Inspire, por sua vez, possuem uma verificação de entrada Centralizada (realizada pela equipe responsável pela plataforma) e Fechada (não disponibiliza a avaliação de RED publicamente). Por fim, as plataformas Edmodo e Amazon Inspire, apesar de serem consideradas fechadas, disponibilizam os critérios de avaliação e seleção de RED publicamente. Na maioria das plataformas analisada destacam-se a ausência de transparência quanto aos critérios adotados para a avaliação de submissão no que tange a aceitação de conteúdo, bem como sobre a própria avaliação. Tal informação muitas vezes está ausente ou se encontra diluída nos Termos de Uso ou de Conteúdo.

Já no segundo momento - após a publicação dos recursos nas plataformas buscamos analisar como eram feitas as avaliações dos RED. Nesse sentido, identificamos a existência de duas frentes: a participação da comunidade de usuários e a avaliação por especialistas vinculados a plataforma. Dessa maneira, primeiro investigamos como era realizada a avaliação feita pelos usuários cadastrados e, pudemos perceber que era amplamente utilizada em quase todas as plataformas analisadas sempre de maneira aberta (avaliação visível) e descentralizada. Com exceção da plataforma REA Ceibal que não possui esse tipo de avaliação.

Posteriormente buscamos compreender como era realizada a avaliação feita pelos especialistas, que, por sua vez, estava presente em quatro plataformas analisadas, Common Sense Education, Curriki, Merlot e Edmodo. Destacamos que a plataforma Common Sense Education conta com uma equipe de avaliadores que preenchem resenhas e análises dos RED e disponibiliza uma página dedicada a explicar a metodologia de avaliação adotada. A plataforma Merlot, adota avaliação por pares e editores, além do uso de selos. Esta plataforma disponibiliza aos usuários os critérios de avaliação dos RED, bem como o desempenho do mesmo em cada um dos critérios. A plataforma Curriki, por sua vez, conta com uma equipe de avaliadores. Já a plataforma 
VIII Congresso Brasileiro de Informática na Educação (CBIE 2019)

Anais do XXX Simpósio Brasileiro de Informática na Educação (SBIE 2019)

Edmodo utiliza a "recomendação dos editores" e não disponibiliza publicamente os critérios para seleção e avaliação de RED.

Dessa maneira, percebemos diferentes possíveis combinações de processos de curadoria nas plataformas analisadas. Sendo elas, (I) avaliação de entrada, avaliação de especialistas e avaliação dos usuários (Edmodo); (II) avaliação de entrada e avaliação dos usuários (OER Commons, Amazon Inspire, Escola Digital e Portal do Professor); (III) avaliação de especialistas e dos usuários (Common Sense Education, Merlot e Curriki) e apenas (IV) avaliação dos usuários (Relia, CK-12, TV Escola e BIOE).

Dada às possibilidades de combinações das informações advindas da análise, percebeu-se a necessidade de organização dos dados. Para isso utilizou-se a abordagem de Hylén (2006), explorada por Cechinel (2017), organizando os processos de curadoria adotadas pelas plataformas nos eixos: aberto versus fechado e centralizado versus descentralizado.

\section{Quadro 2. Organização do processo de curadoria das plataformas na tipificação de Hylén (2006) e Cechinel (2017)}

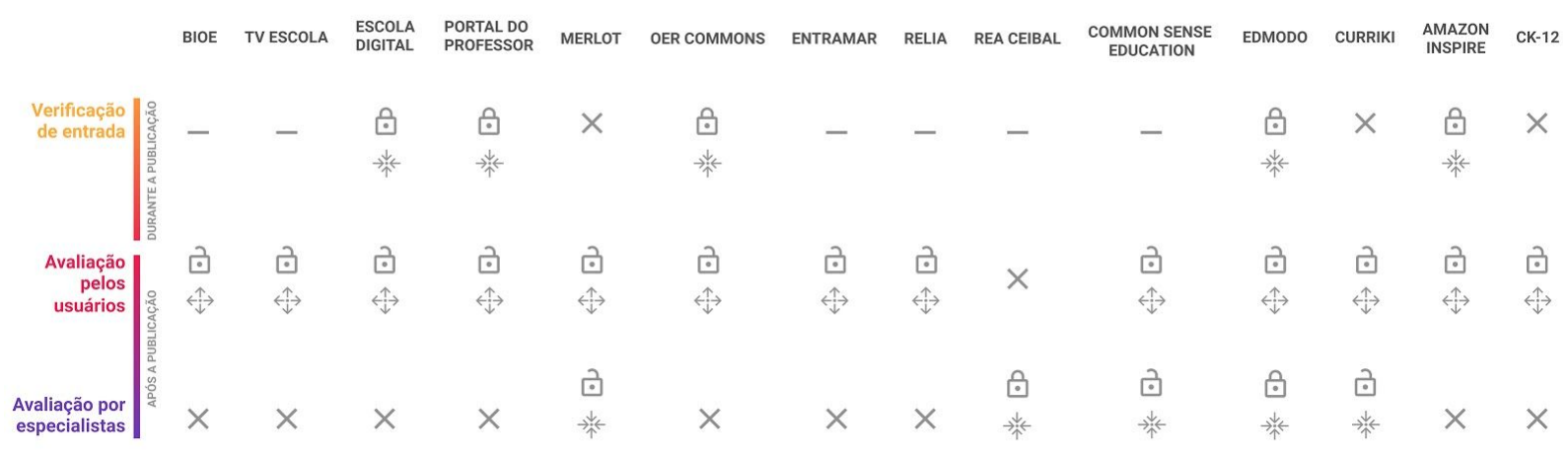

\section{LEGENDA}

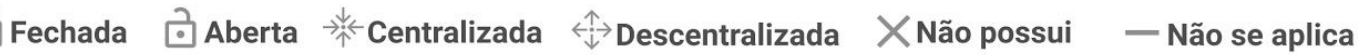

Dessa maneira, compreende-se que o processo de verificação de entrada, quando existe, é geralmente Centralizado. Ou seja, o usuário não possui participação direta no processo. Essa verificação de entrada Centralizada pode ser Aberta ou Fechada na medida em que a plataforma explicita - ou não - os seus critérios de avaliação aos usuários.

Sobre o processo que sucede a publicação do recurso nas plataformas, podemos afirmar que a avaliação feita por usuários está presente em todos as plataformas analisadas, com exceção da plataforma REA Ceibal. Esta avaliação, em todos os casos, é realizada de maneira Aberta.

Ainda sobre a avaliação feita por usuários está expressa nas plataformas das seguintes maneiras: escala de estrelas, comentário, criação de coleções ou favoritos, preenchimento de formulário de avaliação e/ou a combinações destas. Todas as plataformas analisadas que possibilitavam a avaliação pelos usuários adotaram algum deste métodos citados. Destaca-se a plataforma OER Commons que permite aos seus usuários uma avaliação do RED através de "escala de estrelas", bem como a possibilidade de avaliar o recurso de forma mais densa a partir de um formulário guiado e formativo. 
VIII Congresso Brasileiro de Informática na Educação (CBIE 2019)

Anais do XXX Simpósio Brasileiro de Informática na Educação (SBIE 2019)

A partir da revisão bibliográfica e do processo de análise, foi possível elencar critérios essenciais aos dois momentos identificados - durante e após a publicação - que são explicitados no quadro 3, a seguir.

Quadro 3. Sistematização dos critérios

\begin{tabular}{|l|l|l|l|}
\hline \multicolumn{2}{|c|}{ Durante a Publicação } & \multicolumn{2}{c|}{ Após a Publicação } \\
\hline $\begin{array}{c}\text { Verificação de } \\
\text { metadados }\end{array}$ & \multicolumn{1}{|c|}{$\begin{array}{c}\text { Verificação de } \\
\text { entrada }\end{array}$} & $\begin{array}{c}\text { Critérios da avaliação } \\
\text { por especialistas }\end{array}$ & $\begin{array}{c}\text { Critérios da avaliação } \\
\text { pelos usuários }\end{array}$ \\
\hline Etiquetamento & Conduta do Conteúdo & Aspectos Pedagógicos & Aspectos Pedagógicos \\
\cline { 2 - 4 } & Aspectos Técnicos & Qualidade do Conteúdo & Qualidade do Conteúdo \\
\cline { 2 - 4 } & Aspectos Pedagógicos & Qualidade Técnica & Qualidade Técnica \\
\cline { 2 - 4 } & & & Contexto de Uso \\
\hline
\end{tabular}

No decorrer da publicação, elencamos os critérios em dois eixos, sendo eles, Verificação de metadados, onde o etiquetamento diz respeito aos dados iniciais do RED; e a Verificação de entrada, que considera três principais critérios: conduta do conteúdo que é o processo cuidadoso de investigar possíveis conteúdos indevidos; aos aspectos técnicos que verifica possíveis problemas técnicos no RED, por exemplo o funcionamento do som em vídeos ou à legibilidade de uma fotografia, entre outros; e os aspectos pedagógicos presentes no RED.

Após a publicação do recurso, o processo também foi dividido em dois eixos: Critérios de avaliação por especialistas, o qual curam os aspectos pedagógicos, a qualidade do conteúdo apresentado, junto com a qualidade técnica do recurso; e Avaliação pelos usuários que possui as mesmas dimensões de critérios elencada no eixo dos especialistas, e ainda avalia o contexto de uso daquele recurso, por meio de interação e manifestações da comunidade de usuários.

\section{Considerações finais}

Esta pesquisa apresentou o resultado de um estudo exploratório que buscou analisar os processos de curadoria de diferentes plataformas educacionais digitais. Foram analisadas um total de 14 plataformas a partir das especificidades do ciclo de vida dos RED, e da tipificação das dimensões de avaliação de recursos educacionais digitais.

Nesse sentido, percebemos que a concepção de Curadoria Digital compreende e contempla a integração das tecnologias digitais, à gestão ativa (intervenção humana) e as práticas de arquivamento de dados. Entendemos que o processo de Curadoria Digital - com todas suas particularidades e implicações - acontece em momentos diferentes e ao longo de todas as etapas do ciclo de vida de um RED.

Inferimos que quando há curadoria de verificação de entrada, ela geralmente é centralizada, sendo ela aberta ou fechada, à depender da disponibilização da avaliação por parte da Plataforma. Já sobre o processo posterior a publicação do recurso é entendido que a avaliação feita por usuários está presente em grande parte das plataformas analisadas, sendo a avaliação aberta em todos os casos. 
VIII Congresso Brasileiro de Informática na Educação (CBIE 2019)

Anais do XXX Simpósio Brasileiro de Informática na Educação (SBIE 2019)

Portanto, entendemos que o processo de Curadoria Digital se dá em dois momentos - antes e após a publicação de RED. No contexto de uma plataforma de RED vinculado a um órgão público, acreditamos que: a) Durante a Publicação o processo deve ser aberto - possibilitando transparência e orientação aos usuários - e centralizado, por meio de algoritmos visando conferir a existência dos metadados e pela intervenção humana com o intuito de evitar violações aos termos de uso. No segundo momento, b) Após a Publicação, defendemos dois tipos de posturas, conforme o tipo de usuário: aberta e descentralizada, valorizando a participação ativa dos sujeitos envolvidos no processo (sobretudo professores) por meio dos seus comentários e avaliações; e postura aberta e centralizada, por envolver a avaliação e certificação de especialistas.

A utilização das abordagens combinadas implica na constituição de uma rede qualificada de RED, prezando a transparência das avaliações propostas. Por fim, ainda pensando em curadoria digital, é importante que haja dimensões, fluxos e critérios próprios para cada momento do ciclo de vida de um RED, idealizando uma curadoria digital concreta e fundamentada.

\section{Referências}

Abbot, D. (2008) “What is digital curation?”, In: Digital Curation Center.

Barros, N. N. (2014) "Apropriação da curadoria na web por uma empresa de mídia tradicional: um caso de convergência entre narrativa e banco de dados", Dissertação (Mestrado) Universidade Estadual de Campinas, São Paulo.

Beagrie, N. (2019) "Preservation management of digital materials: The Handbook", Digital Preservation Coalition, nov. 2008.Brasil. Ministério da Educação. Situando a curadoria: avaliação e seleção de RED. In: Formação de curadores.

Cechinel, C. (2017) "Modelos de curadoria de recursos educacionais digitais", In: Centro de Inovação Para A Educação Brasileira - Cieb.

Collis, B., \& Strijker, A. (2004). Technology and Human Issues in Reusing Learning Objects. Journal of Interactive Media in Education; May 2004: JIME Special Issue on the Educational Semantic Web, 2004(1). Retrieved from http://jime.open.ac.uk/article/2004-4-collis/187\%3C/div\%3E.

Hylén, J. (2006). Open educational resources: Opportunities and challenges. Proceedings of Open Education, 49-63.

Meurer, H. and Szabluk, D. (2010) "Projeto E: aspectos metodológicos para o desenvolvimento de projetos digit-virtuais”, In: Ação Ergonômica - ERGODESIGN II, v. 5, n. 2 .

Sales, L. F. and Sayão, L. F. (2012) "O impacto da curadoria digital dos dados de pesquisa na comunicação científica", Encontros Bibli: Revista Eletrônica de Biblioteconomia e Ciência da Informação, v. 17, n. esp. 2, p.118-135. 